\title{
Angiomatosis bacilar por Bartonella quintana en un paciente con infección por virus de inmunodeficiencia humana
}

\author{
Patricia Vásquez T., Leonardo Chanqueo C., Patricia García C., Helena Poggi M., \\ Marcela Ferrés G., Marisol Bustos M. y Antonio Piottante B.
}

\section{Bacillary angiomatosis caused by Bartonella quintana in an human immunodeficiency virus positive patient}

We report the first case of bacillary angiomatosis due to Bartonella quintana affecting a Chilean a HIV positive patient in Chile. He was a 27 years old, heterosexual male, indigentman known to be HIV positive serological status known from September, 2003, under irregular medical control. On April, 2005, he presented a progressive abscess in the frontal region and erythematous papules in the extremities, that extended to face, thorax and mucoses, becoming nodular and violaceous lesions. Bacillary angiomatosis diagnosis was initially sustained on account of the clinical manifestations, and was confirmed by serology and Warthin Starry staining from a skin biopsy. The etiological agent was identified as Bartonella quintana through universal RPC performed from a cutaneous nodule to detect 16S rRNA gen. Azithromycin plus ciprofloxacin was started, besides of anti retroviral therapy antiretroviral, with the lesions being progressively disappearing.

Key words: Bacillary angiomatosis, Bartonella quintana infection, bartonelosis in inmunocompromissed patient.

Palabras claves: Angiomatosis bacilar, infección por Bartonella quintana, bartonelosis en inmunocomprometidos.

\section{Introducción}

$\mathrm{L}$ as infecciones producidas por Bartonella sp son conocidas desde la antigüedad en los pueblos aborígenes andinos ${ }^{1} \mathrm{y}$, en la actualidad, se consideran como enfermedades re-emergentes ${ }^{2}$.

El género Bartonella incluye más de 17 especies. La infección es transmitida por varios vectores, incluyendo piojos, pulgas, mosquitos y gatos, los que provocan en el ser humano varios síndromes clínicos ${ }^{3,4}$. Los más frecuentes son: enfermedad por arañazo de gato (EAG) causada por $B$. henselae ${ }^{5}$, enfermedad de Carrión y verruga peruana, por $B$. bacilliformis ${ }^{6}$, fiebre de las trincheras por $B$. quintana ${ }^{7}$, endocarditis y neuroretinitis, por $B$. elizabethae, angiomatosis bacilar y peliosis hepática en hospederos inmunocomprometidos, por $B$. henselae y $B$ quintana, $a^{8,9}$.

Tanto la angiomatosis bacilar como la peliosis hepática bacilar, son diferentes manifestaciones clínicas de un proceso de proliferación vascular secundarias a la infección por el género Bartonella, el que ocurre, principalmente, en pacientes con inmunodeficiencia adquirida $^{10,11}$. En la angiomatosis bacilar, las lesiones se encuentran en la piel, con características inespecíficas (tamaño diverso, únicas o múltiples, cutáneas o subcutáneas), pueden ser pápulas, nódulos o placas, siendo lo más característico una pápula roja purpúrica. También pueden presentarse lesiones mucosas (de localización oral, anal, conjuntival o gastrointestinal), compromiso de hígado, bazo, médula ósea y hueso ${ }^{12,13}$. La peliosis bacilar corresponde a una entidad estrechamente relacionada, que se caracteriza por lesiones angioproliferativas del hígado y bazo ${ }^{14}$.

Notificamos el primer caso de angiomatosis bacilar por B. quintana en un paciente con infección por VIH, diagnosticado en el Centro de Atención de pacientes infectados con VIH/SIDA, del Hospital San Juan de Dios de Santiago, Chile.

\section{Caso clínico}

Varón de 27 años de edad, residente de Santiago, soltero y heterosexual, con diagnóstico de infección por VIH desde septiembre de 2003 y serología confirmatoria efectuada por el laboratorio de referencia del Instituto de Salud Pública (ISP). Fue catalogado en etapa A, nunca se realizó determinación de linfocitos CD4 y tuvo controles irregulares en otra policlínica. Desde hacía 10 años vivía en la calle, dedicado al
Hospital San Juan de Dios Servicio de Medicina Interna Unidad de Infectología. (PVT, LChC, MBM)

Unidad de Anatomía Patológica (APB) Pontificia Universidad Católica de Chile

Laboratorio de Microbiología y Biología Molecular UDA y Servicio de Laboratorios Clínicos (PGC, HPM) Laboratorio Infectología y Virología Molecular (MFG)

Recibido: 02-06-2006 Aceptado: 22-12-2006

Correspondencia a: Leonardo Chanqueo Cornejo Ichanqueo@gmail.com 
Figura 1. Lesiones papulares eritematosas en la zona de la barba y subcutáneas cervicales.

Figura 2. Lesiones papulares violáceas en la región anterior del tórax.

Figura 3. Lesión exofítica costrosa conjuntival en ángulo interno del ojo derecho comercio ambulante y alojaba en residencia para individuos indigentes.

Desde abril de 2005 presentó un aumento de volumen en la región frontal, asociándose, además, lesiones papulares eritematosas en extremidades, por lo
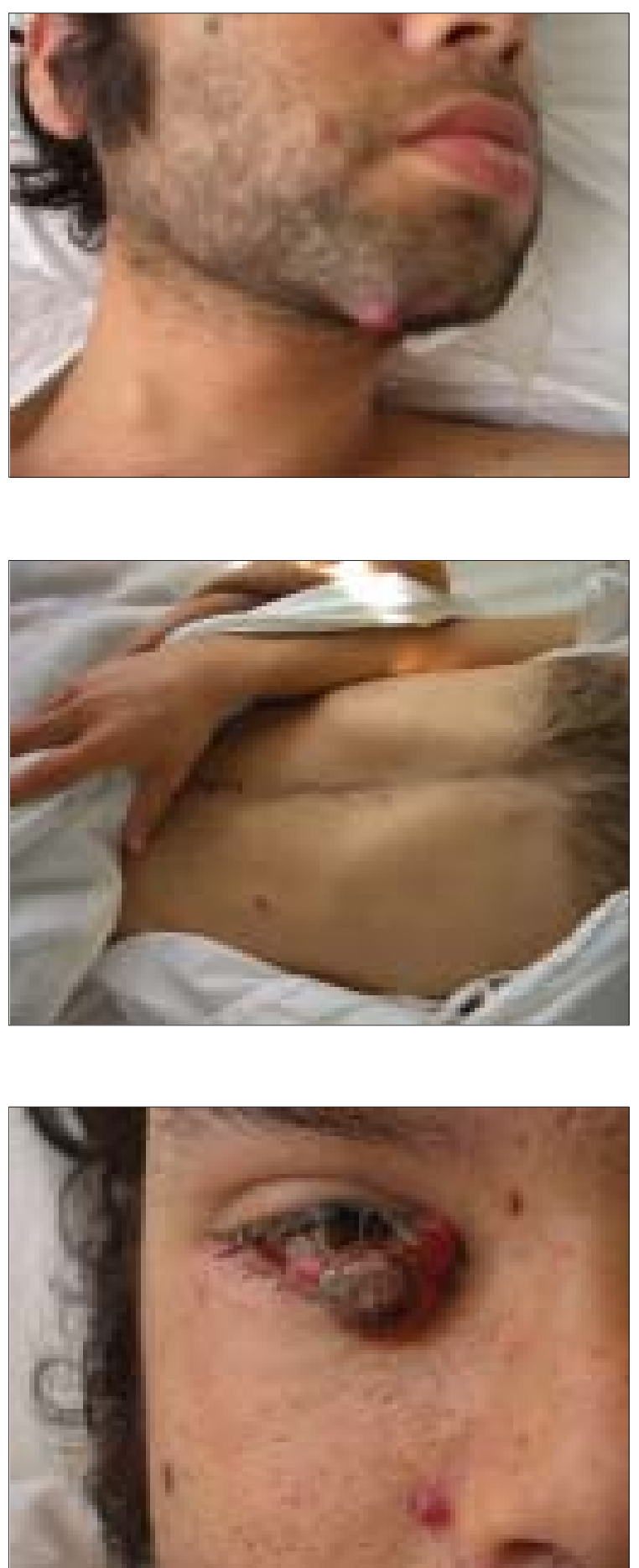

que consultó en policlínica de Infectología-VIH, del Hospital San Juan de Dios, en agosto de 2005. Se observaba un absceso frontal y lesiones de tipo forúnculos en ambas extremidades, siendo enviado a servicio de urgencia para debridamiento del absceso y la obtención de cultivos. Acudió a control después de dos semanas, con progresión de absceso frontal ( 5 x 4 $\mathrm{cm}$ de diámetro), con un área de necrosis central y borde eritematoso extenso, coincidiendo con la aparición de nuevas lesiones papulares eritematosas de 1 a $2 \mathrm{~cm}$. de diámetro en la cara, principalmente, en la zona de la barba (Figura 1), en extremidades inferiores- algunas subcutáneas de consistencia blanda, no dolorosas- y lesiones violáceas en la región anterior del tórax y en las extremidades inferiores (Figura 2). También se apreció compromiso mucoso, con lesiones exofiticas costrosas en el ángulo interno del ojo, de $2 \mathrm{~cm}$ de diámetro, en ambas conjuntivas (Figura 3) y lesiones exofíticas con ulceración central en la mucosa oral, localizadas en la región vestibular del labio inferior, paladar duro y blando (Figura 4).

En el examen físico general se destacó un paciente en malas condiciones higiénicas, pálido, con candidiasis orofaríngea, $\mathrm{T}^{\circ}$ axilar de $37,5^{\circ} \mathrm{C}$, un índice de masa corporal de 18, sin adenopatías regionales. El examen cardiovascular no reveló alteraciones y no se apreció visceromegalia abdominal. No presentaba compromiso neurológico clínico.

Otros antecedentes relevantes que aparecieron en la historia clínica fueron viajes como mochilero a Perú (Tacna) en 2001 y a Bolivia (Ororuro) en el 2003. No tenía mascotas; sin embargo, refirió tener contacto, en forma frecuente, con gatos callejeros de todas las edades, habiendo sido rasguñado ocasionalmente.

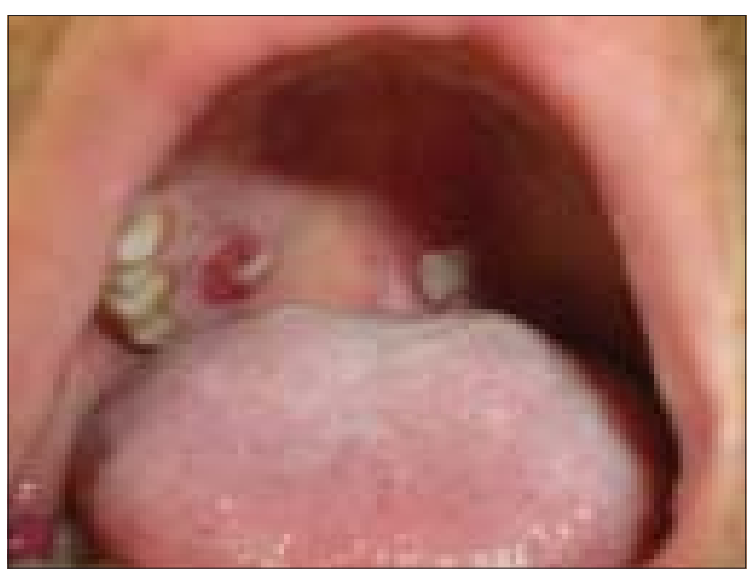

Figura 4. Lesiones exofíticas con ulceración central en mucosa oral (paladar). 
Se decidió, entonces, su hospitalización para realizar estudios. De los exámenes generales destacó un hematocrito de $31 \%$ con VCM 80 fl y CHCM 32\%, leucocitos de 5.700 céls $/ \mathrm{mm}^{3}$, sin desviación a izquierda y plaquetas 310.000 céls $/ \mathrm{mmm}^{3}$, VHS $81 \mathrm{~mm} / \mathrm{h}$, sin alteración de las pruebas de función hepática, con fosfatasa alcalina de 85 U/L y LDH 185 U/L. Del estudio de su patología de base, tenía una carga viral para VIH (Nuclisens HIV-1 QT, Biomérieux) de 130.000 copias ARN viral/ml y un recuento de linfocitos CD4 de 83 céls $/ \mathrm{mm}^{3}$. Los hemocultivos automatizados aeróbicos fueron negativos a los siete días de incubación, el cultivo de secreción y de la biopsia de lesión frontal también fue negativo para bacterias, lo mismo la baciloscopia y el cultivo de micobacterias. El estudio de imágenes realizado mostró: radiografía de tórax normal, ecografía abdominal sin hallazgos patológicos y radiografía de cráneo sin lesiones osteolíticas.

Se envió una muestra de suero para la determinación de anticuerpos IgG para $B$. henselae y $B$. quintana por inmunofluorescencia (Focus $\left.{ }^{\circledR}\right)$ al Laboratorio de Infectología y Virología Molecular de la Pontificia Universidad Católica de Chile, que resultó con títulos $>1 / 256$ para $B$. henselae y $>1: 64$ para $B$. quintana.

Se le efectuó biopsia de la lesión cutánea frontal cuyo informe indicó: piel ulcerada por epitelio escamoso maduro, proliferación lobulillar de vasos de pequeño calibre, abundantes polimorfos nucleares intersticiales, algunos con cariorrexis. La tinción de Warthin Starry demostró estructuras cocobacilares en acúmulos sugerentes de Bartonella sp.

Dada la alta sospecha clínica, después de tomar cultivos y biopsia, se inició tratamiento con azitromicina $500 \mathrm{mg} / \mathrm{d}$ v.o. más ciprofloxacina $500 \mathrm{mg}$ c/12 hs v.o. Se realizó una cirugía de resección de las lesiones exofiticas conjuntivales bilaterales, las que se enviaron a estudio histológico que concluyó: tejido conectivo con epitelio escamoso y cilíndrico hiperplásico, proceso inflamatorio agudo y crónico, abundantes vasos de neoformación, material amorfo intersticial. La tinción de Warthin Starry demostró estructuras sugerentes de Bartonella spp, compatible con angiomatosis bacilar.

Para el diagnóstico molecular se efectuó una biopsia de un nódulo violáceo de la región abdominal anterior y se envió al Laboratorio de Biología Molecular de la Pontificia Universidad Católica de Chile. La extracción del ADN del tejido subcutáneo se realizó utilizando un método comercial (QIAamp Tissue kit, Qiagen $($ )), según las recomendaciones del fabricante. La amplificación del gen que codifica para el 16S ARNr se realizó con partidores universales ${ }^{15}$. Posteriormente, se secuenció el producto amplificado y se evaluó en el analizador genético ABI 310. Para la comparación de la secuencia nucleotídica obtenida con las secuen- cias depositadas en banco de genes Genebank, se utilizó al programa BLAST. Se encontró una homología de $100 \%$ sobre 418 pares de bases depositadas con la especie B. quintana subespecie Toulouse, que se confirmó, posteriormente, por amplificación y secuenciación de los segmentos ITS y 336, que son específicos a nivel de género y de especie, respectivamente.

Previo al alta, se inició terapia antiretroviral con zidovudina, lamivudina y efavirenz con diagnóstico de infección por VIH en etapa B-3 y angiomatosis bacilar por B. quintana. La evolución posterior al inicio de la antibioterapia ha sido satisfactoria, con regresión del compromiso cutáneo y mucoso a los cuatro meses de tratamiento.

\section{Discusión}

La angiomatosis bacilar fue descrita por primera vez en 1983 por Stoler y cols en relación con la epidemia de VIH/SIDA ${ }^{16}$. Se observó que pacientes con infección por VIH avanzada desarrollaban nódulos subcutáneos diseminados, cuyo agente fue caracterizado inicialmente como un organismo rickettsia-like, estrechamente relacionado con Rochalimaea quintana ${ }^{17}$. Posteriormente se identificaron como agentes causales de esta patología a $B$. quintana y B. henselae ${ }^{18}$.

La especie responsable se puede sospechar según los factores de riesgo epidemiológico involucrados y la predilección por algún órgano ${ }^{19}$. Es así como la infección por $B$. quintana se encuentra en pacientes de bajo nivel socio-económico, vagabundos, con alcoholismo crónico y pediculosis corporal (Pediculus humanus $)^{20,21}$ y sus manifestaciones clínicas se asocian, además de angiomatosis bacilar, a compromiso óseo y subcutáneo ${ }^{7,18}$. En cambio, B. henselae tiene un reservorio felino claramente establecido y se asocia a mordeduras o rasguños de gatos $^{22}$ y a picaduras de pulgas portadas por éstos (ectoparásito Ctenocephalides felis $)^{23}$. El compromiso clínico clásico es la EAG clásica, con linfoadenopatías regionales, pudiendo existir compromiso extra-ganglionar ${ }^{24}$ y más relacionado con peliosis hepática ${ }^{7}$.

En este paciente se podría haber sospechado infección por $B$. quintana, dado el nivel socioeconómico y la situación de indigencia, aunque en el examen físico no se encontró ectoparásitos. Sin embargo, tenía el antecedente de contacto frecuente con gatos callejeros que, de acuerdo con datos nacionales, tienen alta seroprevalencia para $B$. henselae ${ }^{25,26}$. Entonces, es fundamental para el diagnóstico etiológico, no sólo el estudio tradicional de la biopsia, sino también la serología y el análisis molecular. 
El estudio anatomo-patológico es vital en estos casos, ya que se deben descartar otras etiologías. En nuestro país, debemos considerar como diagnóstico diferencial más importante, el sarcoma de Kaposi especialmente cuando las lesiones no son tan extensas- y la micobacteriosis. Otras etiologías a considerar son el granuloma piógeno, los linfomas cutáneos, los hemangiomas, los tumores dérmicos y, con menor frecuencia, la verruga peruana y las micosis tropicales ${ }^{12,27}$.

Para acercarse al diagnóstico de especie se han descrito la serología específica (IgM y/o IgG cuantitativas) y la reacción de polimerasa en cadena (RPC) específica para Bartonella, que es el método de elección en la identificación directa de la bacteria desde sangre o tejidos ${ }^{19}$.

La serología tiene una sensibilidad en el diagnóstico de EAG de $90 \%$ y en endocarditis bacteriana, de $97 \%{ }^{28}$. En este paciente se obtuvo una serología positiva para $B$. henselae y para $B$. quintana, siendo los títulos para $B$. henselae mayores que para $B$. quinta$n a$. Sin embargo, es conocido que existen reacciones cruzadas entre las diferentes especies del género Bartonella, como también con otros microorganismos como Chlamydia sp, Coxiella burnetii y Anaplasma phagocytophilum $^{29,30}$. La detección de ADN bacteriano y posterior identificación de especie en las lesiones presenta una sensibilidad de hasta $100 \%$ en muestras de tejidos en pacientes con angiomatosis bacilar ${ }^{18,28}$. En el caso de este paciente, la RPC universal, con posterior secuenciación, permitió la identificación de B. quintana.

Al igual que lo descrito en la literatura, los cultivos fueron negativos, lo que se explica porque el aislamiento de Bartonella sp en humanos, por métodos microbiológicos clásicos, es complejo y de bajo rendimiento $^{31,32}$.

En Chile, se desconoce el espectro de la enfermedad por Bartonella sp, siendo lo más reconocido la EAG en su forma clásica y/o complicada, con un claro rol de los gatos en la transmisión. Sin embargo, debemos considerar otras formas de presentación, como las manifestaciones clínicas en hospederos inmunocomprometidos, caracterizadas por cuadros más gra- ves p. ej: síndrome febril prolongado ${ }^{33}$, endocarditis, bacteriemia, angiomatosis bacilar y peliosis hepática. Esta bacteria fue recientemente descrita, por primera vez en Chile, en una paciente con endocarditis infecciosa por métodos moleculares ${ }^{34}$.

Este caso clínico constituye el primer reporte confirmatorio en Chile de angiomatosis bacilar por Bartonella quintana en un paciente infectado por VIH y demuestra la utilidad de los métodos de biología molecular para la identificación del agente etiológico.

Estos hallazgos deben hacer que el médico clínico sospeche este agente en pacientes con cuadros clínicos sugerentes y motivar la búsqueda tanto de ésta como de otras especies y sus reservorios animales.

\section{Agradecimientos}

Las fotografías fueron tomadas con el debido consentimiento informado del paciente.

\section{Resumen}

Reportamos el primer caso de angiomatosis bacilar por Bartonella quintana en un paciente con infección por VIH en nuestro país. Este corresponde a un hombre de 27 años, heterosexual, indigente, seropositivo para VIH conocido desde septiembre de 2003, en control irregular. En abril de 2005, el paciente desarrolló un aumento progresivo de volumen en la región frontal y aparición de pápulas eritematosas en las extremidades, que luego se extendieron a la cara, tórax y mucosas, tornándose nodulares y violáceas. El diagnóstico de angiomatosis bacilar se planteó inicialmente por el cuadro clínico del paciente, siendo confirmado por serología y tinción de Warthin Starry positiva en la biopsia de piel. El agente causal se identificó como Bartonella quintana por RPC universal para el gen del $16 \mathrm{~S} \mathrm{ARNr}$ de un nódulo cutáneo. Se inició terapia antimicrobiana con azitromicina y ciprofloxacina, además de terapia antiretroviral, con desaparición de las lesiones en forma progresiva.

\section{Referencias}

1.- Maguiña C. Las viejas y nuevas bartonellas: una enfermedad emergente mundial. Acta Méd Peruana 2001; 18 (3): 122-32.

2.- Higgins R. Emerging or re-emerging bacterial zoonotic diseases: bartonellosis, leptospirosis, Lyme borreliosis, plague. Rev Sci Tech Off Int Epiz 2004; 23: 569-81.

3.- Breitschwerdt E, Kordick D. Bartonella infection in animals: carriership, reservoir potential, pathogenicity, and zoonotic potential for human infection. Clin Microbiol Rev 2000; 13: 428-38.

4.- Blanco J, Raoult D. Enfermedades producidas por Bartonella spp. Enferm Infecc Microbiol Clin 2005; 23: 313-9.

5.- Abarca K, Vial P, Rivera M, García C, Oddo D, Prado $\mathrm{P}$, et al. Infección por Bartonella henselae en pacientes inmunocompetentes: enfermedad por arañazo de gato. Rev Méd Chile; 1996; 124: 1341-9.

6.- Maguiña C, Cok J, Gilman R, Osores F, Tello A. Estudio prospectivo de la verruga peruana recurrente. Dermatol Per 2003; 13: $189-94$.

7.- Foucault C, Brouqui P, Raoult D. Bartonella quintana characteristics and clinical management. Emerg Infect Dis 2006; 
12:217-23.

8.- Slater L N, Welch D F, Min K W. Rochalimaea henselae causes bacillary angiomatosis and peliosis hepatis. Arch Intern Med 1992; 152: 602-6.

9.- Gasquet S, Maurin M, Brouqui P, Lepidi H, Raoult D. Bacillary angiomatosis in immunocompromised patients. AIDS 1998; 12: $1793-803$.

10.- Cockerell C, Whitlow M, Webster G, Friedman-Kien A. Epithelioid angiomatosis: a distinct vascular disorder in patients with the acquired immunodeficiency syndrome or AIDS-related complex. Lancet 1987; 2 (8560): 654-6.

11.- Tappero J, Mohle J, Koehler J, Swaminathan B, Berger T, LeBoit $\mathrm{P}$, et al. The epidemiology of bacillary angiomatosis and bacillary peliosis. JAMA 1993; 269: 770-5.

12.- Velho P, Souza E, Cintra M, Mariotto A, Machado A. Angiomatose bacilar: revisão da literatura e documentação iconográfica. An bras Dermatol 2003; 78: 601-9.

13.- Gazineo J, Trope B, Maceira J, May S, Coelho J, Lambert J, et al. Bacillary angiomatosis: description of 13 cases reported in five reference centers for AIDS treatment in Rio de Janeiro, Brazil. Rev Inst Med Trop S Paulo 2001; 43: 1-6.

14.- Perkocha L, Geaghan S, Yen B, Nishimura S, Chan S, García-Kennedy R, et al. Clinical and pathological features of bacillary peliosis hepatis in association with human inmunodeficiency virus infection. N Engl J Med 1990; 323: 1581-6.

15.- Baker G, Smith J, Cowan D. Review and re-analysis of domain-specific $16 \mathrm{~S}$ primers. J Microbiol Methods 2003;55: 541-55.

16.- Stoler M, Bonfiglio T, Steigbigel R, Pereira M. An atypical subcutaneous infection associated with AIDS. Am J Clin Pathol 1983; 80: 714-8.

17.- Relman D, Loutit J, Schmidt T, Falkow S, Tompkins L. The agent of bacillary angiomatosis. An approach to the identification of uncultured pathogens. N

Engl J Med 1990; 323: 1573-80.

18.- Koehler J, Sánchez M, Garrido C, Whitfeld M, Chen F, Berger T, et al. Molecular epidemiology of bartonella infections in patients with bacillary angiomatosis-peliosis. N Engl J Med 1997; 337: $1876-83$

19.- Anderson B, Neuman M. Bartonella spp as emerging human pathogens. Clin Microbiol Rev 1997; 2: 203-19.

20.- Jackson L, Spach D. Emergence of Bartonella quintana infection among homeless persons. Emerg Infect Dis 1996; 2: 141-4.

21.- Brouqui P, Lascola B, Roux V, Raoult D. Chronic Bartonella quintana bacteremia in homeless patients. N Engl J Med 1999; 340: 184-9.

22.- Chang C, Chomel B, Kasten R, Tappero J, Sánchez M, Koehler J. Molecular epidemiology of Bartonella henselae infection in human immunodeficiency virusinfected patients and their cat contacts, using pulsed-field gel electrophoresis and genotyping. J Infect Dis 2002; 186: 1733-9.

23.- Chomel B, Kasten R, Floyd-Hawkins K, Chi B, Yamamoto K, Roberts-Wilson J, et al. Experimental transmission of Bartonella henselae by the cat flea. J Clin Microbiol 1996; 34: 1952-6.

24.- Wolff E, Muñoz M, Zapata C, Ledermann W. Enfermedad por arañazo de gato complicada con compromiso sistémico, osteomielitis osteovertebral y absceso paravertebral. Rev Chil Infect 2000; 17: 332-9.

25.- Zaror L, Ernst S, Navarrete M, Ballesteros A, Doroscheck D, Ferres M, et al. Detección serológica de Bartonella henselae en gatos en la ciudad de Valdivia, Chile. Arch Med Vet 2002; 1: 103-10.

26.- Ferrés M, Abarca K, Godoy P, García P, Palavecino E, Méndez G et al. Presencia de Bartonella henselae en gatos: cuantificación del reservorio natural y riesgo de exposición humana de esta zoonosis en Chile. Rev Méd Chile 2005; 133: 1465-71.

27.- LeBoit P, Berger T, Egbert B, Beckstead J, Yen T, Stoler M. Bacillary angiomatosis: the histopathology and differential diagnosis of a pseudoneoplastic infection in patients with HIV disease. Am J Surg Pathol 1989; 13: 909-20.

28.- La Scola B, Raoult D. Culture of Bartonella quintana and Bartonella henselae from human samples: a 5-year experience (1993 to 1998). J Clin Microbiol 1999; 37: 1899905.

29.- La Scola B, Raoult D. Serological cross-reactions between $B$. quintana, $B$. henselae, and C. burnetii. J Clin Microbiol 1996; 34: 2270-74.

30.- Maurin M, Eb F, Etienne J, Raoult D. Serological cross-reactions between Bartonella and Chlamydia species: Implications for diagnosis. J Clin Microbiol 1997; 35: 2283-7.

31.- Doern G. Detection of selected fastidious bacteria. Clin Infect Dis 2000; 30: 166-73.

32.- Clarridge J, Raich T, Pirwani D, Simon B, Tsai L, Rodrigues-Barradas C, et al. Strategy to detect and identify Bartonella specie in routine clinical laboratory yields Bartonella henselae from human immunodeficiency virus positive patient and unique Bartonella strain from his cat. J Clin Microbiol 1995; 33: 2107-13.

33.- Koehler J, Sánchez M, Tye S, GarridoRowland C, Chen F, Maurer T, et al. Prevalence of Bartonella infection among human immunodeficiency virus-infected patients with fever. Clin Infect Dis 2003; 37: 559-66.

34.- García P, Poggi H, Lagos M, Romeo E, Ferrés M, Zalaquet R, et al. Bartonella quintana: primer caso identificado en Chile. Libro de Resúmenes XXII Congreso Chileno de Infectología, Puerto Varas, Chile, 26-29 de octubre de 2005. Resumen p13, pág 50 . 